University of Nebraska - Lincoln

DigitalCommons@University of Nebraska - Lincoln

Timothy J. Gay Publications

Research Papers in Physics and Astronomy

March 1979

\title{
Temperature dependence of alignment production in He I by beam-foil excitation
}

Timothy J. Gay

University of Nebraska - Lincoln, tgay1@unl.edu

H. G. Berry

University of Chicago, Chicago, Illinois

Follow this and additional works at: https://digitalcommons.unl.edu/physicsgay

Part of the Physics Commons

Gay, Timothy J. and Berry, H. G., "Temperature dependence of alignment production in He I by beam-foil excitation" (1979). Timothy J. Gay Publications. 6.

https://digitalcommons.unl.edu/physicsgay/6

This Article is brought to you for free and open access by the Research Papers in Physics and Astronomy at DigitalCommons@University of Nebraska - Lincoln. It has been accepted for inclusion in Timothy J. Gay Publications by an authorized administrator of DigitalCommons@University of Nebraska - Lincoln. 
Phys. Rev. A 19, 952 - 961 (1979) [Issue 3 - March 1979]

\title{
Temperature dependence of alignment production in He I by beam-foil excitation
}

\author{
T. J. Gay and H. G. Berry \\ Department of Physics, University of Chicago, Chicago, Illinois 60637 and Argonne National \\ Laboratory, Argonne, Illinois 60639
}

Received 6 September 1978

We have measured the dependence upon target-foil temperature of the linear polarization fraction $(M / I)$ of the $2 s^{1} S-3 p^{1} P, 5016$ - $\AA$ transition in He I for ion energies between 60 and 180 $\mathrm{keV}$. The thin carbon exciter foils were heated externally by Nichrome resistance elements. The measurements of Hight et al. are duplicated; the energy and current dependencies of $M / I$ are the same, assuming correspondence between beam heating and external heating. We also observe that $\gamma$, the number of secondary electrons produced per incident ion, decreases with increasing foil temperature. These two effects, in conjunction, offer a plausible explanation for the variation of polarization with beam-current density. The temperature of the foil is shown to depend on beam current to the one-fourth power, indicating that radiation is the primary energy-loss mechanism.

(C)1979 The American Physical Society

URL: http://link.aps.org/abstract/PRA/v19/p952

DOI: 10.1103/PhysRevA.19.952 


\title{
Temperature dependence of alignment production in He I by beam-foil excitation
}

\author{
T. J. Gay and H. G. Berry \\ Department of Physics, University of Chicago, Chicago, Illinois 60637 \\ and Argonne National Laboratory, Argonne, Illinois 60639
}

(Received 6 September 1978)

\begin{abstract}
We have measured the dependence upon target-foil temperature of the linear polarization fraction $(M / I)$ of the $2 s^{1} S-3 p{ }^{1} P, 5016-\AA$ transition in $\mathrm{He}_{1}$ for ion energies between 60 and $180 \mathrm{keV}$. The thin carbon exciter foils were heated externally by Nichrome resistance elements. The measurements of Hight et al. are duplicated; the energy and current dependencies of $M / I$ are the same; assuming correspondence between beam heating and external heating. We also observe that $\gamma$, the number of secondary electrons produced per incident ion, decreases with increasing foil temperature. These two effects, in conjunction, offer a plausible explanation for the variation of polarization with beam-current density. The temperature of the foil is shown to depend on beam current to the one-fourth power, indicating that radiation is the primary energy-loss mechanism.
\end{abstract}

\section{INTRODUCTION}

In recent studies of the interaction of fast ions with thin carbon foils, Hight et al. ${ }^{1}$ have measured the electronic alignment produced in excited neutral helium. They found that the alignment of the $3{ }^{1} P$ state oscillates as a function of beam velocity and also varies with beam current density. The variation of alignment with beam current density $\Delta A_{0}^{c} / \Delta J$ also oscillates as a function of beam velocity. In this paper we analyze the possible effects on the electronic alignment of the foil temperature and, in turn, of the secondary electrons produced by the ion moving through the solid. We present measurements which explain some of the basic features of the observed alignment variations and discuss other factors contributing to the production of electronic alignment.

It has been shown ${ }^{2,3}$ previously that the number of back-scattered secondary electrons produced when fast ions bombard solid targets is dependent on target temperature, and Sternglass ${ }^{4}$ has explained these results qualitatively. Hence we may expect that secondary electron production may also vary with the foil temperature in fast ion collisions. Therefore, we have performed two experiments attempting to relate the secondary electron flux to the production of alignment in the fast ion beam. We have measured the alignment of the $3 p{ }^{1} P$, He $I$ state at beam energies between 60 and $180 \mathrm{keV}$ as a function of the foil temperature. Second, we have shown that the secondary electron flux varies as a function of the foil temperature for the same ion beam energies. The results show that the alignment variations observed by Hight et al. ${ }^{1}$ are due to changes in the foil temperature. The alignment appears to depend on the secondary electron flux, but this is not definitely proven by the experiments.
In Sec. II, we describe the experimental arrangement for our measurements of the temperature dependence of the optical polarization and secondary electron flux. Results of these experiments are presented in Sec. III. In Sec. IV we discuss possible explanations for our observations and the implications which these observations have in terms of existing theories of the interaction of fast ions with thin solid foils.

During the course of this work, we have developed a new method for measuring the temperature of thin carbon exciter foils. A detailed description of this method is presented in an Appendix.

\section{EXPERIMENT}

Experimental work was carried out with the University of Chicago's 250-keV linear accelerator. The experimental arrangement is shown in Fig. 1. The $\mathrm{He}^{+}$beam from the accelerator passed through a 6.4- $\mathrm{mm}$ diameter tantalum collimator and then through the carbon foil located downbeam. Beam dispersion was such that its diameter increased by roughly $1 \mathrm{~mm}$ by the time it reached the foil, so that the beam aperture was completely filled.

The temperature of the carbon exciter foils was measured using an Ircon $300 \mathrm{~L}$ bolometer, having a spectral range of 2.0-2.6 $\mu$ (see Appendix). The bolometer was sighted through a quartz window on the side of the chamber, the line of sight being $39.5^{\circ}$ from the foil normal. The carbon foils were supported and externally heated by an assembly consisting of two machined glass ceramic plates sandwiching a length of 26 gauge nichrome wire, bent around 3 beam apertures. The thickness of the heater assembly required that one side of the holes be beveled outward (see Fig. 1) in order to maintain an unobstructed line of sight for the bolometer. The wire was attached to two binding posts 


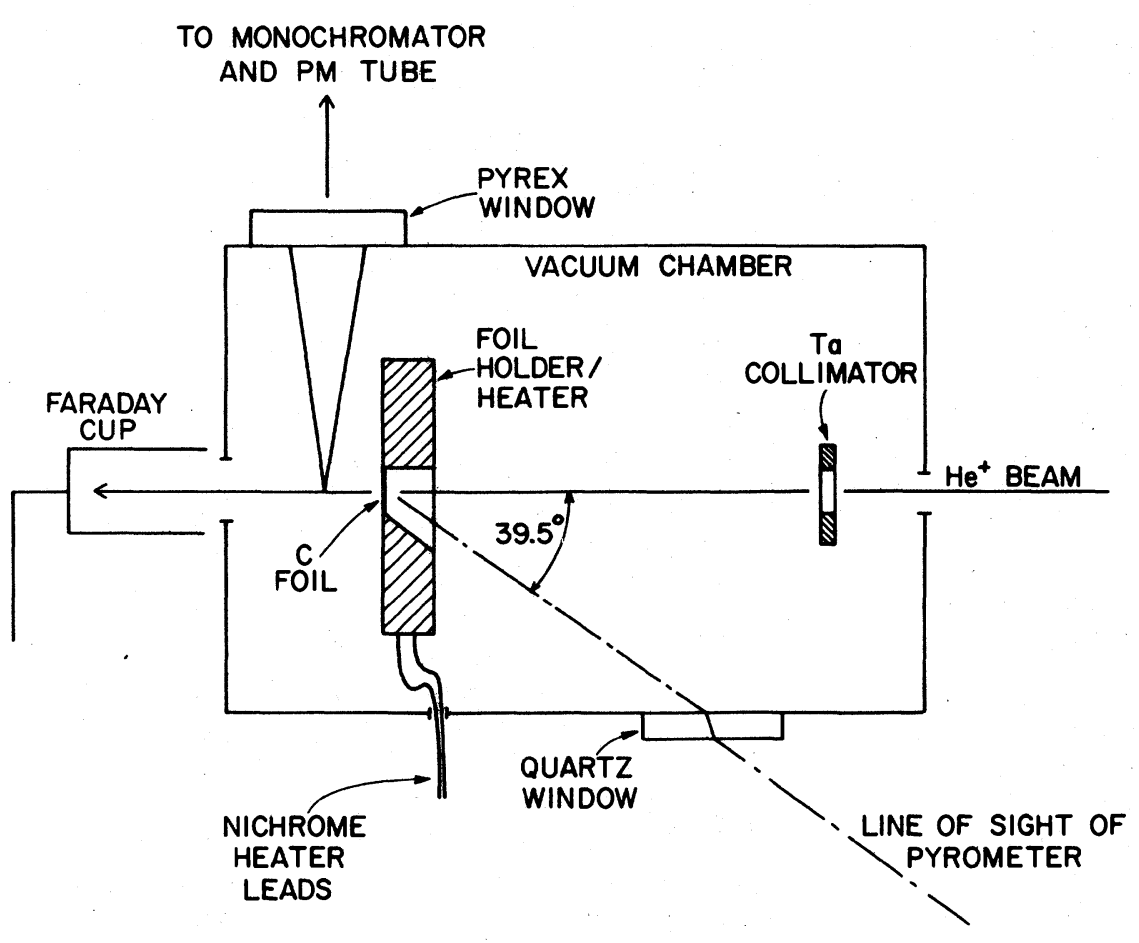

FIG. 1. Experimental arrangement for polarization measurements from top of vacuum chamber. at either end of the plates, which held the assembly together and acted as feed-ins for the heater current. With a maximum current of $\sim 6 \mathrm{~A}$, the heater was able to produce foil temperatures in excess of $950^{\circ} \mathrm{K}$. The ceramic plates were coated with carbon black in order to reduce the background light in the chamber due to glowing of the nichrome when it was hot.

The chamber pressure during these experiments was nominally $5 \times 10^{-6}$ Torr. When the nichrome filament was hot, however, the pressure rose quickly and then fell slowly to a constant level as the foil holder outgassed. At the highest heater current, the pressure was about $9 \times 10^{-6}$ Torr.

Using the optical detection system described by Berry et $a l .{ }^{5}$ we measured the linear polarization fraction Stokes parameter $(M / I)$ for the $2 s{ }^{1} S$ $-3 p{ }^{1} P, 5016-\AA$ transition in He $I$. Beam energies were varied between 60 and $180 \mathrm{keV}$, with the carbon exciter foils being perpendicular to the ion beam in all cases. For this geometry and atomic transition, $M / I$ is related to the Fano-Macek ${ }^{6}$ alignment parameter $A_{0}^{\text {col }}$ by the equation

$$
M / I=-3 A_{0}^{\mathrm{col}} /\left(2-A_{0}^{\mathrm{col}}\right),
$$

where

$$
A_{0}^{\mathrm{col}}=\left\langle 3 L_{\|}^{2}-L^{2}\right\rangle /\left\langle L^{2}\right\rangle,
$$

$L_{11}^{2}$ being the component of angular momentum along the beam axis.

For $M / I$ measurements, light yield was normal- ized to beam current collected in the Faraday cup. Because the cup was not shielded from stray secondary electrons, we determined the actual ion flux by measuring current with an empty foil holder in the beam. That we are not measuring actual beam current during the run is unimportant as far normalization is concerned. There is a small correction due to the fact that secondary electron current is not strictly proportional to ion beam current (see Sec. III). In practice, this correction is negligible. Ideally, the experiment should be performed in the limit as beam current vanishes. Adequate statistics, however, demanded that beam currents of at least $0.5 \mu \mathrm{A}$ be used. At the beginning of each run, the temperature of the foil was measured with external heating only, and also with the beam passing through the foil. The current was adjusted so that the difference between these two temperatures was always less that $15^{\circ} \mathrm{C}$. During the $M / I$ versus temperature runs, care was taken to avoid beam "hot spots" or small areas of high current density, which would have produced artifically high values of $M / I$ (due to the current effect of Hight et al.). These were visible as pinpricks of enhanced luminescence on the quartz beam stop at the end of the Faraday cup, and were eliminated by adjusting acceleration, extraction, and focussing voltages.

The secondary electron experiment was performed with foils mounted on Al holders attached to a wheel that rotated them into the beam. An 
electric field plate, $2 \mathrm{~cm} \times 3 \mathrm{~cm}$ (extending $3 \mathrm{~cm}$ downbeam), connected to a high voltage power supply was placed $1 \mathrm{~cm}$ above the grounded foil holders. Secondary electron production was monitored by reading the current between the foil holder and plate when the potential difference was $4000 \mathrm{~V}$.

\section{RESULTS}

In order to demonstrate an equivalence between temperature and beam current effects, it was first necessary to obtain the relationship between current density $J$ through the foil and the foil temperature $T$. The temperature versus current relationship at a beam energy of $127 \mathrm{keV}$ is shown in Fig. 2. Calculations of the relative effectiveness of heat dispersion by conduction, convection, and radiation show that for $10 \mu \mathrm{g} / \mathrm{cm}^{2}$ foils and a chamber temperature of $293^{\circ} \mathrm{K}$, for foil temperatures above $530^{\circ} \mathrm{K}, 90 \%$ or more of the foil's heat loss is due to radiation. As a result, we expect foil temperature to go as $J^{1 / 4}$. This is what is observed; if we fit the data of Fig. 2 with the functional form $a+b T^{4}$ we obtain the curve shown, with a zero-current intercept of $308 \pm 100^{\circ} \mathrm{K}$, in good agreement with the data. The error bars are conservative. Discrepancies from the exact $T^{4}$ curve may be due to other energy loss mechanisms such as secondary electron emission, sputtering, or an underestimation of the importance of conduction and convection.

$M / I$ was measured as a function of foil temperature at energies ranging from 60 to $180 \mathrm{keV}$. The beam current was kept at about $1 \mu \mathrm{A}$. The first point at all energies was taken without external heating, thus providing us with a base temperature of $\sim 620^{\circ} \mathrm{K}$. Current through the Nichrome heater was then increased in steps until maximum temperatures in the vicinity of $950^{\circ} \mathrm{K}$ were reached. Dark count varied from 6-8 s without heating to $10-12 \mathrm{~s}$ with maximum heater current. Signal count rates, including dark count, were nominally 60-90 s. Each $M / I$ point is the average of 4 or 5 sets of 8 pairs of readings, each pair giving an $M / I$ value. Each set represents about 16000 counts. The standard deviation of the sets was always close to $1 \%$ in $M / I$. Since each $M / I$ determination was based on roughly the same number of raw counts, this $1 \%$ figure was adopted as the error estimate for all data points. A typical slope, at $122 \mathrm{keV}$ is shown in Fig. 3. The time required to take data points was about $\frac{1}{2} \mathrm{~h}$. This effectively eliminated any possible short-term time-dependent effects.

Fitting a straight line to these points gives a slope $S_{T}=\Delta(M / I) / \Delta T$, which we have determined as a function of energy. These results are shown in Fig. 4. The error bars of the points were assigned by the fitting routine at each energy. A comparison of these results with those of Hight et al. is possible by assuming, for simplicity, a linear relationship between beam current and foil temperature instead of the actual $T^{4}$ dependence. This is a good approximation in the temperature range of our experiments $\left(T>600^{\circ} \mathrm{K}\right)$ and is certainly valid considering the size of the error bars in Fig. 2. $T$ is now proportional to $-(d E / d x)^{1 / 4} I$, where $-(d E / d x)$ is the stopping power for $\mathrm{He}^{+}$on

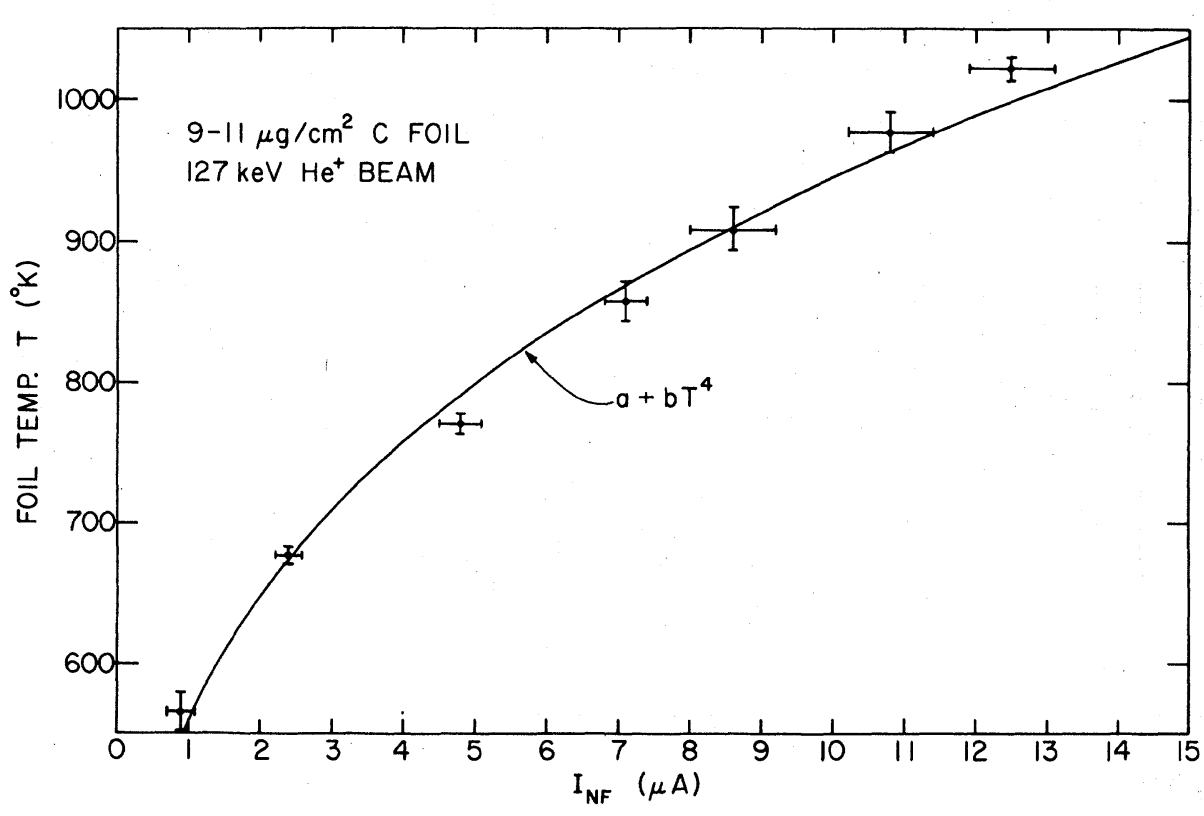

FIG. 2. Foil temperature $\left({ }^{\circ} \mathrm{K}\right)$ vs beam current $(\mu \mathrm{A}) ; 6.4-\mathrm{mm}$ diameter beam aperture. Measurements were taken with foils ranging from 9 to 11 $\mu \mathrm{g} / \mathrm{cm}^{2}$ with a $127-\mathrm{keV} \mathrm{He}^{+}$ beam. Solid line is leastsquares fit for $I=a+b T^{4}$. $I_{N F}=$ beam current with no foil in place. 


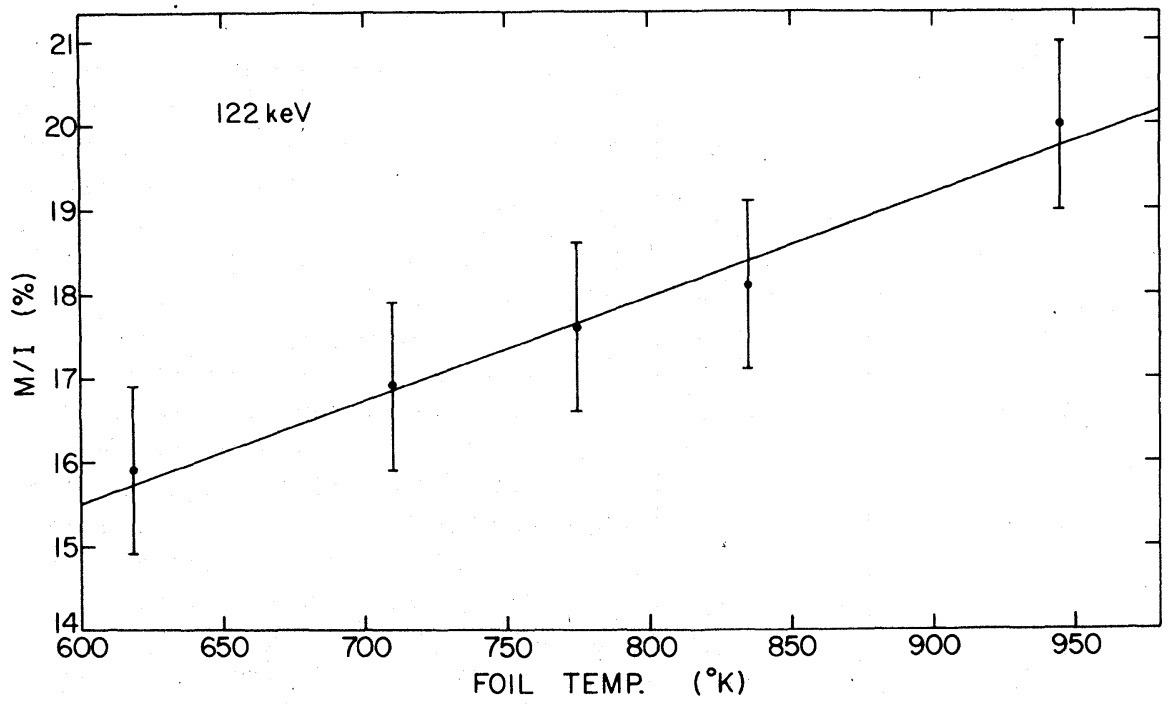

FIG. 3. Linear polarization $(M / I)$ of the $2 s^{1} S$ $-3 p^{1} P 5016 \AA$ transition in He $I$ vs foil temperature. Ion energy $=122 \mathrm{keV}$.

$C$ (Ref. 7) and is monotonically increasing with energy for our energy range. By fitting a straight line to the top six data points of Fig. 2 and dividing Hight's $S_{J}$ values by its slope times $\{[d E / d x(E) /$ $[d E / d x(E=127 \mathrm{keV}]\}^{1 / 4}$ we get the equivalent value of $S_{T}$ at energy $E$. For comparison, we have drawn a smooth line through Hight's data and transposed it to the equivalent $S_{T}$ curve to obtain the line in Fig. 4. Our ability to duplicate with $S_{T}$ the energy dependence of $S_{J}$ is strong evidence that the current dependence is in fact a temperature dependence.

$M / I$ versus beam energy for the cases of no heating and maximum heating are shown in Fig. 5 . The equivalent current data is also shown. Error bars on these points are again about $1 \%$ in $M / I$. The good agreement between the high-current results of Hight et al. and our temperature data taken at $\sim 920^{\circ} \mathrm{K}$ is perhaps the best demonstration of the equivalence between current and temperature effects.

In the secondary-electron experiment, we measured $\gamma$, the ratio of the ion beam current to the electron current measured at the field plate. The foil temperature was changed by varying the beam current. The magnitude of $\gamma$ varied from 8 to 13 ,

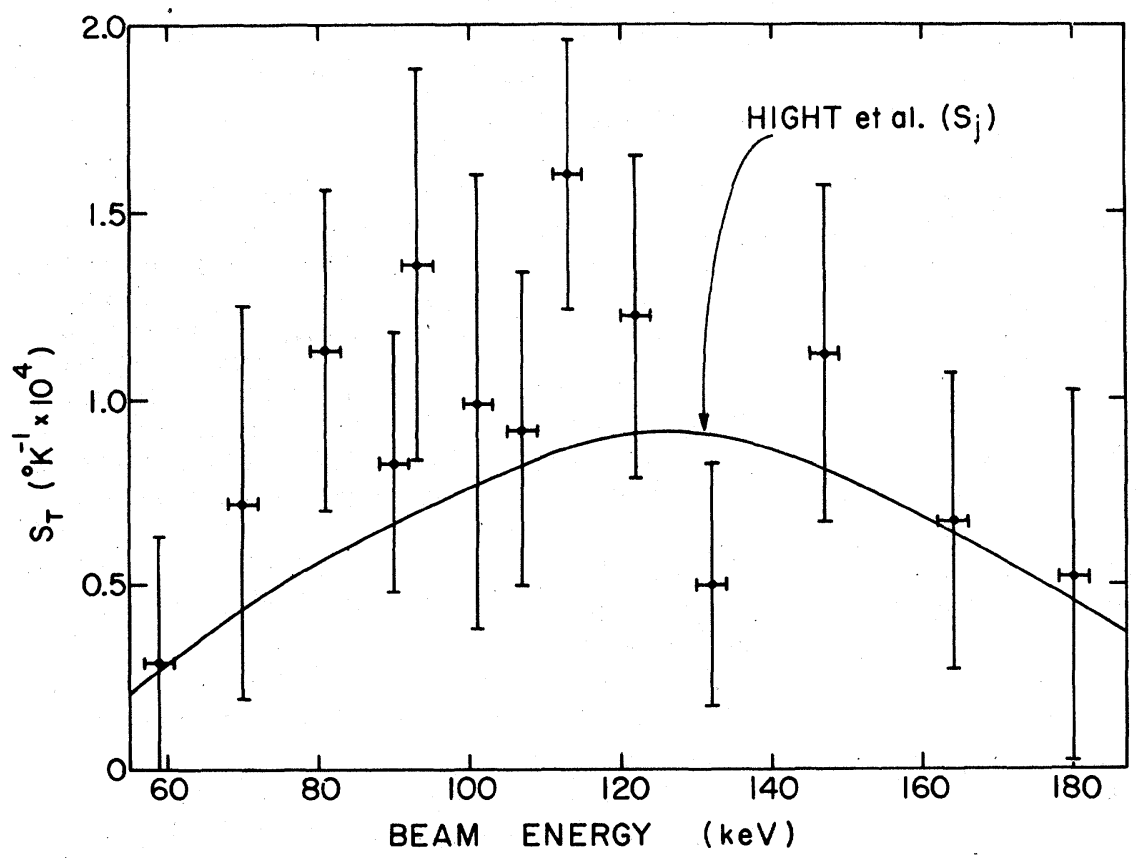

FIG. 4. $S_{T}(\Delta(M / I) / \Delta T)$ vs beam energy (5016 $\AA$ ). The line corresponds to the equivalent beam current data of Hight et al. (Ref. 1; See text). 


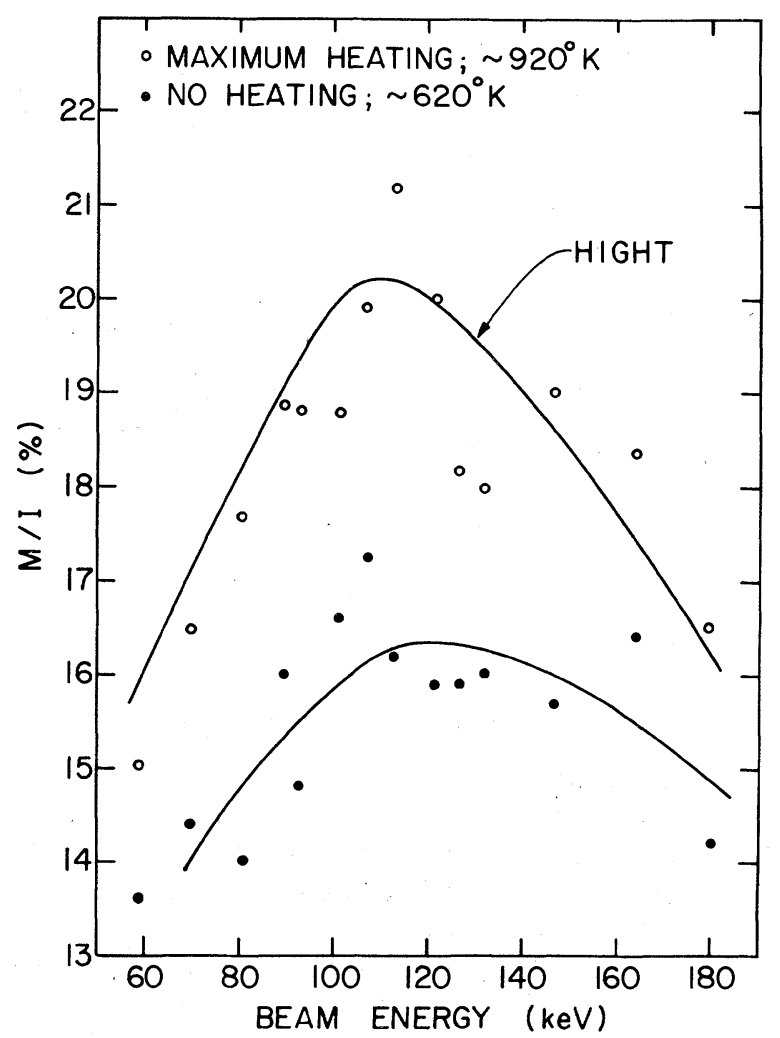

FIG. 5. Linear polarization fraction (5016 $\AA$ ) vs beam energy. The upper points were taken for foil temperatures of $920 \pm 20^{\circ} \mathrm{K}$. The upper line represents the data of Hight $e t$ al. taken with beam currents which produce foil temperatures of $900-950^{\circ} \mathrm{K}\left(\sim 29 \mu \mathrm{A} / \mathrm{cm}^{2}\right)$. The lower points were taken with no heating. The line through them is drawn for comparison with the high temperature (current) data.

a result which agrees qualitatively with Meckbach's ${ }^{8}$ observations, although our method was by no means a quantitative way to achieve results for the secondary yield. Our experiment was unable to differentiate between angle of emission, electron energy, or the side of the foil from which the secondaries emerged. However, our relative electron yields should be more accurate, and of the order of $\pm 10 \%$. In Fig. 6 we show the variation of electron flux with foil temperature at a beam energy of $175 \mathrm{keV}$. A marked decrease results from increasing the temperature. A least-squares fit to the data gives $S_{\gamma}=(\Delta \gamma / \Delta T)$, which is plotted versus beam energy in Fig. 7. Similar decreases in secondary emission (seen as an increase in Faraday cup current) were observed when the foil was heated externally during the course of the polarization measurements. The electron current due to thermionic emission here is negligible. The thermionic current from the foil at $900^{\circ} \mathrm{K}$ is $<10^{-15}$ $\mu \mathrm{A}$.

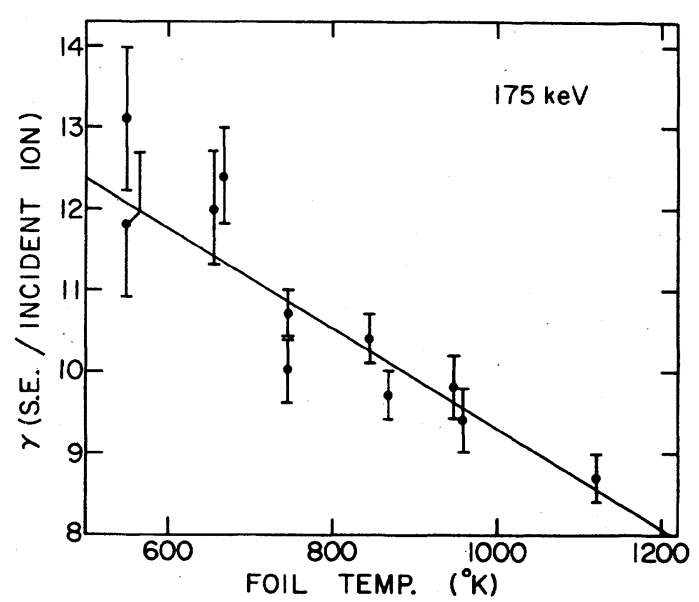

FIG. 6. $\gamma[\equiv$ (secondary-electron current/beam current)] vs foil temperature at $175 \mathrm{keV} . S_{\gamma}$ is the slope of the fitted line.

\section{DISCUSSION}

The present work was undertaken to explain some of the puzzling features which were observed by Hight et al. in their investigation of beam-foil collision induced alignment of atomic states, specifically the $3 p^{1} \mathrm{P}$ state of $\mathrm{HeI}$. A brief review of their work follows. The linear polarization fraction $M / I$ of the $2 s{ }^{1} S-3 P$ transition $(5015 \AA$ ) as a function of beam energy is shown in Fig. 8(a). The data below $0.4 \mathrm{MeV}$ is that of Hight et al.; the higher energy data was taken by the present authors at Argonne National Laboratory using the Dynamitron accelerator. The experimental arrangement and the data reduction procedures were identical to those used by Hight et al. The crosses represent points taken with a beam current density of $30 \mu \mathrm{A} /$ $\mathrm{cm}^{2}$, corresponding to foil temperatures ranging from 900 to $1000^{\circ} \mathrm{K}$, depending on beam energy. The dots represent $M / I$ values extrapolated to zero beam current (see Ref. 1, Fig. 1). The two interesting features here are the semi-oscillatory behavior of $M / I$ with energy, and the previously mentioned current dependence. Figure 8(b) shows $S_{J}$ as a function of beam energy. Oscillatory behavior occurs here also, with two definite maxima. It is interesting to note that the first two maxima of $S_{J}$ occur at the same energies as the first maximum and minimum of $M / I$.

This work attempts to partially explain the current dependence of $M / I$. Our experiments show that this effect is due to the temperature change of the foil caused by beam heating. The equivalence between external heating and beam heating is demonstrated graphically in Figs. 4 and 5. We are, within experimental error, able to quantitatively reproduce both the dependence of $M / I$ on 


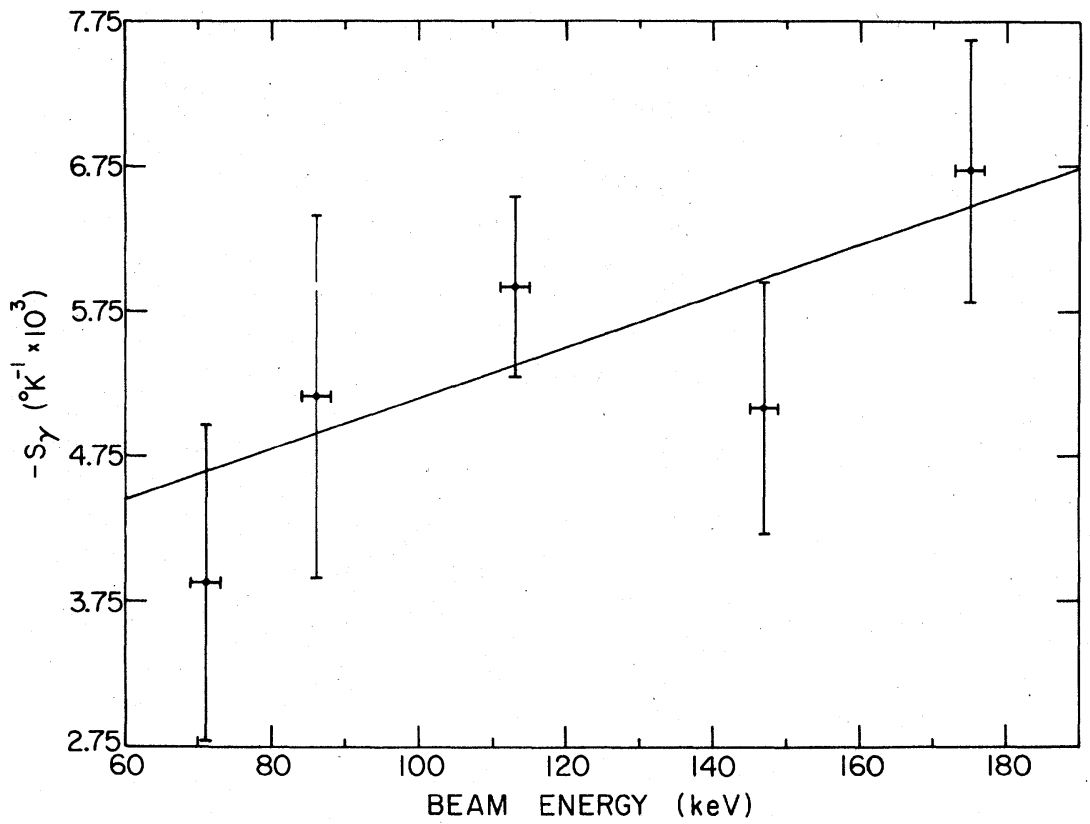

FIG. 7. $S_{\gamma} \equiv(\Delta \gamma / \Delta T)$ vs beam energy. Increase corresponds to increase in foil stopping power at higher energies.

current (temperature), and $S_{J}$ 's dependence on energy. We now propose a model to explain the mechanism whereby temperature change affects the electronic states of ions emerging from the foil.

One readily observable change which occurs when the foil is heated is that $\gamma$, the number of secondary electrons produced per incident ion, decreases. Previously, this had been observed only for thick solid targets. ${ }^{2,3}$ The theory for temperature reduction of $\gamma$ has been developed by Sternglass. ${ }^{4}$ Secondary electrons are produced in a solid in one of two ways. "Primary" secondaries are produced in glancing collisions between the ion and an electron of a target atom. If, however, a head-on collision occurs between the incident ion and this electron, a $\delta$ ray, or "knock-on" electron is produced, which can have velocities 3 or 4 times that of the ion. This fast electron in turn produces slower secondary electrons through glancing collisions. The number of secondaries produced which actually makes it to the foil surface is governed by their mean free path in the solid for undergoing inelastic collisions. If an electron loses too much energy through collisions it will not be able to surmount the surface potential barrier and escape. Heating the foil increases the density of the "phonon gas" through which the electrons must diffuse, i.e., their mean free path is shortened. As a result, $\gamma$ decreases at higher temperatures. If this model holds in our situation, we expect that $\gamma$ will vary with temperature as does the electrical conductivity of a solid. Following Sternglass, we expect

$$
\gamma\left(T_{1}\right) / \gamma\left(T_{2}\right)=1+\beta T_{2} / 1+\beta T_{2},
$$

where $\beta$ is analogous to the temperature coefficient of resistivity. Using the data from Fig. 6 we obtain a $\beta$ of $1.3 \times 10^{-3}{ }^{\circ} \mathrm{C}^{-1}$. Temperature coefficients
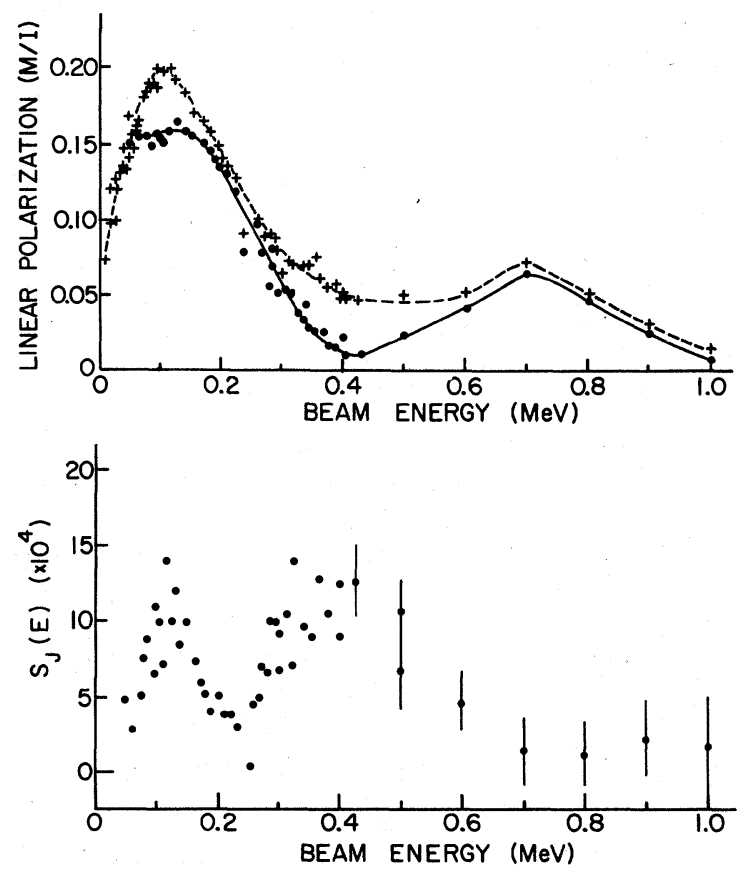

FIG. 8. (a) $M / I$ (5016 $\AA$ ) vs beam energy. Dashed line is for beam current of $30 \mu \mathrm{A} / \mathrm{cm}^{2}$. Solid line is for $M / I$ extrapolated to $O \mu A / \mathrm{cm}^{2}$. (b) $S_{J} \equiv(\Delta M / I / J) \mathrm{vs}$ beam energy. Data of Hight et al. and previously unpublished work by Berry and Gay. 
for most metals are between $5 \times 10^{-4}$ and $5 \times 10^{-3}$ ${ }^{\circ} \mathrm{C}^{-1}$. Hence our results are consistent with a phonon gas model.

"Primary" secondaries, while having roughly the same initial velocity as the ion (10-12 eV energy losses for secondary production are typical), lose energy through collision and will, in general, trail behind the ion as it leaves the surface. Secondaries produced by collision with $\delta$ rays, however, tend to reach the surface before the ion. The number of electrons trailing and preceding the ion should be roughly equal, because equal amounts of energy go into each production mechanism, as shown by Bethe ${ }^{9}$ and Bohr. ${ }^{10}$ The escape depth for secondaries, i.e., the greatest depth at which a secondary can be produced and still escape the surface is, at most, $30-40 \AA{ }^{4}$ Since production is peaked in the forward direction, the large majority of secondaries, while not necessarily having velocities parallel to the ion beam, will emerge no more than 10-15 $\AA$ from its track.

These considerations lead to a simple explanation of the temperature dependence of the alignment, or $M / I$. As the foil temperature increases, the number of secondary electrons produced by each ion decreases. These electrons surround the ion as it leaves the surface, at distances of $\sim 10 \AA$, creating a random electric field in the rest frame of the ion, rapdily varying in time. This field will have peak strengths on the order of $5 \times 10^{7} \mathrm{~V} / \mathrm{cm}$, and will completely mix any electronic states already produced in the ion. This mixing will reduce the degree of alignment (or anisotropy) in the electron cloud surrounding the ion, hence reducing the polarization observed. For higher temperatures and fewer electrons surrounding the emerging ion, we expect higher degrees of anisotropy in the excited states.

For this model to make sense, there must be some other mechanism for producing the anisotropic state. This mechanism could possibly be the static surface fields discussed by $\mathrm{Eck}^{11}$ and Lombardi. ${ }^{12}$ However, the oscillatory structure as a function of beam energy of both the alignment and its temperature-beam-current dependence $S_{T}$ or $S_{J}$, point to a dynamic time-dependent surface field, creating alignment by Stark mixing of different $l$ sublevels within a given $n$ state. ${ }^{13,14}$ Such a time-dependent field develops from the polarization wake first postulated by Bohr. ${ }^{10}$ In this model extended by Neufeld and Ritchie $\mathrm{e}^{15}$ and by Vager and Gemmell, ${ }^{16}$ the moving ion passing through the solid induces a polarization wake which oscillates both in distance from the ion and in time, with the plasma frequency of the solid. The wake also decays in time, at a rate determined by the plasma damping constant. The induced wake produces strong electric fields $\left(\sim 10^{8} \mathrm{~V} / \mathrm{cm}\right)$ which extend about $10 \AA$ outside the foil surface as the ion leaves the foil. The field seen by the ion depends on its energy (velocity) and the plasma frequency of the solid. The length of time during which the field and the ion interact is determined by the time constant for plasmon damping and the velocity of the particle. A possible test of this model is to probe the energy dependence of alignment as a function of foil material, i.e., as a function of plasma frequency and plasma damping constant. Some initial work in this respect has been done ${ }^{17}$ but not over a large enough energy range to provide a test.

We should note that the influence of secondary electrons on the polarization has not been rigorously proved. Such proof would be difficult to obtain experimentally. Secondary-electron emission varies little for different target materials, ${ }^{4}$ and for a given material there is no way to change $\gamma$ without changing temperature also.

Our secondary electron model, while explaining why polarization increases with temperature, fails to explain the oscillatory behavior of $S_{J}$. One would naively expect $S_{J}$ to vary in a similar manner to $S_{\gamma}$, but this is not the case.

The variation of $S_{\gamma}$ is easily explained by considering the stopping power for $\mathrm{He}^{+}$on $C$ as a function of energy. $\gamma$, and hence $S_{\gamma}$, are proportional to stopping power. Since $-(d E / d x)$ increases monotonically with beam energy below $750 \mathrm{keV}$, we expect a similar rise in $-S_{\gamma}$ for our energy range. If we fit a straight line to the data of Fig. 7 and compare the percentage change in $S_{\gamma}$ with the same change in stopping power between 70 and $180 \mathrm{keV},{ }^{7}$ we obtain $0.30 \pm 0.15$ and 0.37 , respectively; the results agree to within the error of the fit.

The failure of our model to explain the behavior of $S_{J}$ with beam energy leads us to consider other possible mechanisms for the current-temperature effect. One possible explanation is that by heating the foil, we are changing its structure, which in turn affects the outgoing ion. Kakinoki et al., ${ }^{18}$ Kupperman et al., ${ }^{19}$ and Devenji et al..$^{20}$ have all observed structural changes in carbon films which they heated above $800^{\circ} \mathrm{K}$. These changes were not reversible, however, indicating that a structural effect cannot explain our results, which are. Another explanation is that we are changing the surface characteristics of the foil by thermal desorption of contaminants. We have observed that $M / I$ values do not change for ambient chamber pressures ranging from $5 \times 10^{-7}$ Torr to $10^{-5}$ with and without cold trapping, a result which tends to discount this possibility. However, experiments at ultra-high vacuum with absolutely clean surfaces are needed before it can be rejected comple- 
tely.

Two other observations we made shed some light on the secondary electron model. During the secondary-electron experiments, we measured several values of $M / I$ at different beam currents and energies. These values were independent of external fields (up to $\sim 4000 \mathrm{~V} / \mathrm{cm}$ ). This implies that if the secondaries are in fact affecting polarization, they must be doing so close to the surface, before they can be swept away by an external field. We also measured the ratio of intensities of the $3 p^{3} P-4 d^{3} D \mathrm{HeI}, 4471 \AA$ and the $n=3-4$ HeII, $4686 \AA$ transitions for different beam currents at a beam energy of $120 \mathrm{keV}$ to see if the charge state ratio of the emerging ions varies with temperature. There was no observable change within statistics between 600 and $960{ }^{\circ} \mathrm{K}$. This result implies that the secondary electrons are not being picked up as the ion emerges. This conclusion is supported by the absolute intensitiy measurements of Gardiner, ${ }^{21}$ although his temperature measurements, as discussed in the Appendix, may be inaccurate.

\section{CONCLUSIONS}

We have shown that the current dependence of the linear polarization fraction $M / I$ of the $5016 \AA$, $2 s{ }^{1} S-3 p^{1} P$ transition in $\mathrm{He} I$ is due to the temperature increase in the foil caused by beam heating. In addition, we find that as the foil temperature increases, the number of secondary electrons produced by each ion decreases. We suggest that the reduction in electrons surrounding the ion as it leaves the foil surface leads to the increased alignment $(M / I)$ at higher temperature. The energy dependencies of $M / I$ and its variation with temperature-beam current are not explained by the monotonic change of $S_{\gamma}(d \gamma / d T)$ with energy. We suggest that the energy variations are produced by a time dependent surface electric field produced at least partially by the decaying polarization wake in the solid.

\section{ACKNOWLEDGMENTS}

The authors would like to thank C. Greene for several helpful discussions. We are especially indebted to $\mathrm{B}$. DeSerio for the development of the microprocessor system which was used in much of the data taking.

This work was supported in part by a grant from the Research Corporation, the NSF, and the U.S. Department of Energy, Division of Chemical Sciences. We thank Professor J. A. Simpson for providing time on the LASR accelerator, and $\mathrm{S}$. Tejero for much helpful technical assistance.

\section{APPENDIX}

Temperature measurement

The temperature of the carbon exciter foil was measured using an infrared optical pyrometer; specifically an Ircon 300L bolometer, which has a spectral range of 2.0-2.6 $\mu$. Other techniques have been used for measuring temperatures of thin foils. Yntema ${ }^{22}$ and Whitmell et al. ${ }^{23}$ have used a furnace surrounding their foils for heating and assume that the temperature of the foil is equal to that of the furnace. As we will show later, this is a poor assumption except at high temperatures. Gardiner ${ }^{21}$ places a thermocouple in close proximity to the foil and wrongly assumes that the temperature he thus measures corresponds to the foil temperature. He fails to observe the considerable foil temperature change due to ion beam heating because the low thermal conductivity of the thin foil allows a large temperature gradient between it and the foil holder.

Temperature monitoring with an infrared optical pyrometer is advantageous because it provides instantaneous, local readings of foil temperature which, again considering the extremely small thermal conductivity of the foils, appears to be important.

The disadvantage with an optical measurement of the temperature is that it requires a knowledge of the emissivity of the foil. The emissivity is a function of the foil thickness, of the observation angle and (except for a gray body) of the wavelength. Our experimental geometry (see Fig. 1) limited our observation angle to $39.5 \pm 2.0^{\circ}$ from the foil normal. The emissivity of a body is given by

$$
\epsilon_{\theta, \lambda}=1-T_{\theta, \lambda}-R_{\theta, \lambda},
$$

where $T$ and $R$ are the transmissivity and reflectivity of that body for a given angle of observation and wavelength. Alternatively, one can use the expression

$$
\epsilon_{\theta, \lambda}=1-T_{\theta, \lambda}^{*}-R_{\theta, \lambda}^{*} \text {. }
$$

The starred quantities refer to the apparent transmissivity and reflectivity. In any simple optical experiment these are the directly measured quantities. Due to multiple reflections at the surfaces the apparent reflectivity is somewhat higher, and the apparent transmissivity somewhat lower than the true values. In order to determine the emissivities of our foils, we mounted test samples of varying thicknesses on Al holders with 6.4- $\mathrm{mm}$ apertures. The apparent transmissivities of the foils as a function of thickness were then measured. We first sighted the pyrometer through a blank aperture on a smooth piece of metal held 


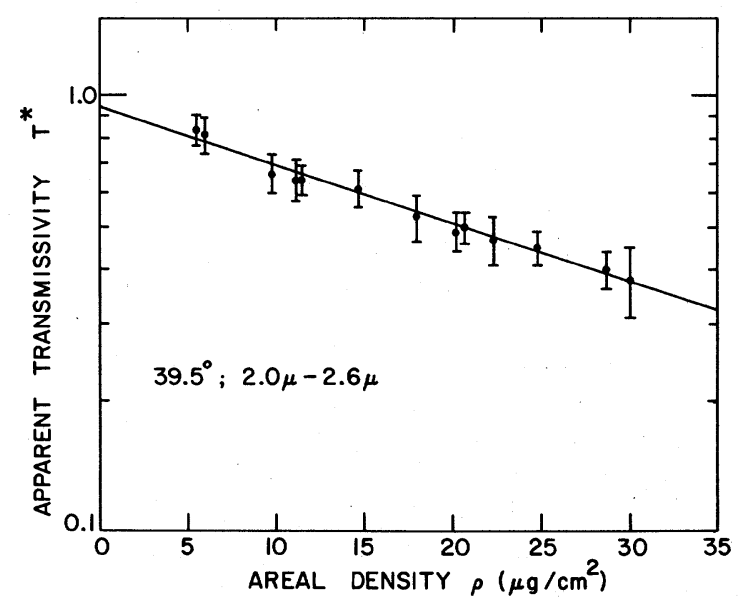

FIG. 9. Apparent transmissivity of carbon foils for radiation between 2.0 and $2.6 \mu$ vs foil areal density. Foil normals tilted $39.5 \pm 1^{\circ}$ to incident radiation. Thickness error bars not shown.

at about $425^{\circ} \mathrm{C}$. The blank was replaced by a foil sample and the ratio of intensities was measured, giving the apparent transmissivity. The plane of the foil was held at $39.5 \pm 1^{\circ}$ relative to the line of sight of the pyrometer. The results of these measurements are shown in Fig. 9. Several foils were used for each thickness in order to account for changes in emissivity due to wrinkling of the surface. The observed apparent transmissivities for different foils of the same thickness group were consistent to an experimental precision for a given measurement of $\pm 10 \%$. Foil thicknesses were those specified by the manufacturer with a quoted error of $\pm 1 \mu \mathrm{g} / \mathrm{cm}^{2}$ or $10 \%$, whichever was bigger. McMahon $^{24}$ has shown that for partially trans- parent reflecting bodies, the apparent transmissivity is given by

$$
T^{*}=T(1-R)^{2} /\left(1-R^{2} T^{2}\right),
$$

where $T$ and $R$ are the true transmissivity and reflectivity of the object. Since for a thin film $T$ can be assumed to vary as $e^{-\lambda t}$, where $\lambda$ is the body's absorption coefficient and $t$ is its thickness, we can write

$$
T^{*}=1-R / 1+R \text { at } t=0 .
$$

Fitting a straight line to the data in Fig. 9 we find that its zero-thickness intercept gives a true reflectivity of $0.03 \pm 0.03$. The fact that the reflectivity is so small justifies our assumption that we could fit a straight line to our data; the second term in the denominator of (A3) becomes negligible so $T^{*}$ simply equals $T$ times a constant. It should be noted that $R$ is independent of thickness. Knowing $R$ and $T^{*}$ versus thickness, we can calculate $T$ versus thickness using (A3). Then, from Eq. (A1), we obtain $\epsilon$ as a function of thickness. These results are shown in Fig. 10. The foils used in our experiment all had areal densities of greater than $9.5 \mu \mathrm{g} / \mathrm{cm}^{-2}$ and less than $11 \mu \mathrm{g} / \mathrm{cm}^{-2}$. For all temperature measurements we assumed the foils had an emissivity of $0.29 \pm 0.10$. While determination of the emissivity for a specific thickness would in theory only require two measurements ( $R^{*}$ and $\left.T^{*}\right)$, in practice measurements of $R^{*}$ are difficult to make and often yield unreliable results, in some cases differing by as much as $100 \%$ for the same foil.

It is reasonable to expect the emissivity to depend on foil structure. During high-temperature runs (see discussion in Sec. III) the foil structure almost certainly changes to some extent, but no

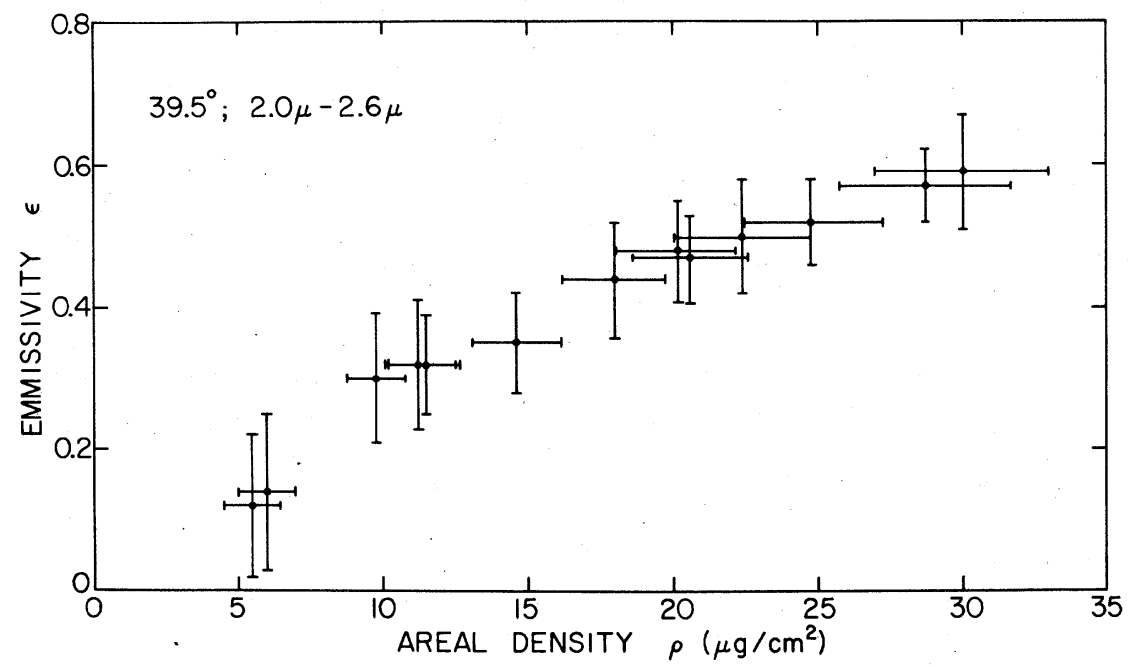

FIG. 10. Emissivity of carbon foils vs areal density $(2.0-2.6 \mu)$. Foil normals tilted $39.5 \pm 1^{\circ}$ to incident radiation. 
corresponding changes in emissivity were observed. This was evidenced by the fact that temperature readings for a given heating condition were highly reproducible, being independent of past heating suffered by the foil. This could only happen if the structural changes are reversible, which is not the case, or if the emissivity does not depend on the foil structure. Foil thickening due to cracking of hydrocarbons at the surface also causes changes in the emissivity, but temperature corrections from this effect are negligible.

During alignment measurements the pyrometer was sighted through a quartz window in the side of the chamber. Due to the small size of the window, and absorption in the quartz itself, radiation from the foil was diminished by a factor of $0.6 \pm 0.1$. This factor, when multiplied by foil emissivity gave an "effective emissivity" of $0.17 \pm 0.07$. This number' was used in all temperature measurements.
The pyrometer measured the temperature of one small area $\left(\sim 3 \mathrm{~mm}^{2}\right)$ of the foil located to one side of the center of the beam aperture. However, the beam diameter at the foil was somewhat larger than the $6.4-\mathrm{mm}$ foil diameter. As a result, the foil area was completely filled with beam and it is a good approximation to say that the spacial variation of foil temperature was negligible.

Even small beam currents create relatively high foil temperatures. $0.9 \mu \mathrm{A}$ over a $6.4-\mathrm{mm}$ diameter aperture is sufficient to raise the foil from room temperature to $575^{\circ} \mathrm{K}$ (see Fig. 2). With a beam current of $12.5 \mu \mathrm{A}$, we reached a temperature of about $1025^{\circ} \mathrm{K}$. Note that at high temperature, for example $1000^{\circ} \mathrm{K}$, the same beam increase of $0.9 \mu \mathrm{A}$ only raises the temperature by about $20^{\circ}$. This implies that while the "furnace method", used by Yntema ${ }^{22}$ to measure temperatures is probably good above $800-900^{\circ}$, it will certainly give poor accuracy below that.
${ }^{1}$ R. D. Hight, R. M. Schectman, H. G. Berry, G. Gabrielse, and T. J. Gay Phys. Rev. A 16, 1805 (1977).

${ }^{2}$ M. L. Oliphant, Pro c. R. Soc. Lond. A 127, 373 (1930).

${ }^{3}$ J. S. Allen, Phys. Rev. 55, 336 (1939).

${ }^{4}$ E. J. Sternglass, Phys. Rev. 108, 1 (1957).

${ }^{5}$ H. G. Berry, G. Gabrielse, and A.E.Livingston, Appl. Opt. 163200 (1977).

${ }^{6}$ U. Fano and J. H. Macek, Rev. Mod. Phys. 45, 553 (1973).

${ }^{7}$ L. C. Northcliffe and R. F. Schilling, Nucl. Data Tables A7, 233 (1970).

${ }^{8}$ W. Meckbach, in Beam Foil Spectroscopy, edited by I. A. Sellin and D. J. Pegg (Plenum, New York, 1975) Vol. 2 .

${ }^{9}$ H. A. Bethe, Ann. Physik 5, 325 (1930).

${ }^{10}$ N. Bohr, K. Dan. Vidensk. Selsk., Mat-Fys. Medd. 18, No. 8 (1948).

${ }^{11}$ T. G. Eck, Phys. Rev。 Lett. 33,1055 (1974)。

${ }^{12}$ M. Lombardi, Phys。 Rev. Lett。 35, 1172 (1975).

${ }^{13}$ M. Lombardi, M. Giroud, and J.H. Macek, Phys. Rev. A 11,1114 (1974).
${ }^{14} \mathrm{E}$. Kupfer and H. Winter, Z. Phys. A285, 3 (1978).

${ }^{15} \mathrm{~J}$. Neufeld and R. H. Ritchie, Phys。 Rev. $\underline{98}, 1632$ (1955).

${ }^{16}$ Z. Vager and D. S. Gemmell, Phys. Rev. Lett. 37 , 1352 (1976).

${ }^{17} \mathrm{H} . \mathrm{G}$. Berry, G. Gabrielse, T. J. Gay, and A. E。 Livingston, Phys. Scr。16, 99 (1977).

${ }^{18}$ J. Kakinoki, K. Katada and T. Hanawa; Acta Crystallogr. 13, 448 (1960).

${ }^{19}$ D. S. Kupperman, C. K. Chau, and H. Weinstock, Carbon 11, 171 (1973).

${ }^{20}$ A. Devenyi, A. Ghevegin, A. Belu, and G. Korony, Second International Conference on Conduction in Low Mobility Materials (Eilat, Israel, 1971) (unpublished) p. 217.

${ }^{21}$ R. B. Gardiner, Radiat. Eff. 35, 151 (1978).

${ }^{22} \mathrm{~J}$. L. Yntema, Nucl. Instrum. Methods 113, 605 (1973)。

${ }^{23}$ D. S. Whitmell, B. H. Armitage, D. R. Porter, and A. T. G. Ferguson, paper presented at the International Conference for Technology of High Energy Accelerators, Daresbury, England (1973) (unpublished).

${ }^{24}$ H. O. McMahon, J. Opt. Soc. Am.40, 376 (1950). 